\title{
COMPORTAMENTO LOCOMOTOR DE CRIANÇAS DE DOIS A SEIS ANOS EM AMBIENTE COMPLEXO
}

\section{LOCOMOTOR BEHAVIOR OF CHILDREN FROM TWO TO SIX YEARS OLD OVER UNEVEN TERRAIN}

Teixeira-Arroyo C, Feitosa EA, Gobbi LTB. Comportamento locomotor de crianças de dois a seis anos em ambiente complexo. Rev Bras Crescimento Desenvolv Hum.2009;19(1): 78-88.

\section{Resumo:}

O comportamento locomotor de 25 crianças, na faixa etária de dois a seis anos, foi observado durante o andar em circuito com obstáculos, sob iluminação normal e reduzida. Os participantes foram instruídos a percorrer o circuito andando o mais rápido possível evitando o contato com os obstáculos. Duas variáveis dependentes foram coletadas: taxa de sucesso e tempo total para percorrer o circuito. As ANOVAs two-way (5 grupo-etário) x (2 iluminação), com medidas repetidas no último fator revelaram apenas efeito principal de grupo para a taxa de sucesso $\left(\mathrm{F}_{4,20}=9,975 ; \mathrm{p}=0,001\right)$, menor para as crianças de 2 anos em relação aos demais grupos, e para o tempo para realizar a tarefa $\left(\mathrm{F}_{4,20}=3,136 ; \mathrm{p}=0,037\right)$, maior para as crianças de 2 anos em relação às de 4 e 6 anos. Análises de Regressão Múltipla (stepwise) revelaram que: idade cronológica, grupo etário, tempo de experiência de andar e tempo gasto para percorrer o circuito predisseram $75,3 \%$ da taxa de sucesso; a taxa de sucesso foi a única variável que significativamente predisse o tempo despendido $\left(\mathrm{R}^{2}=0,372 ; \mathrm{p}=0,001\right)$. Estes resultados permitiram concluir que variáveis desenvolvimentais interferem no comportamento locomotor no circuito com obstáculos e que os níveis de iluminação não foram suficientes para causar perturbação na realização da tarefa.

Palavras-chave: crescimento e desenvolvimento; percepção; locomoção.

1 UNESP - Universidade Estadual Paulista - Campus Rio Claro, Laboratório de Estudos da Postura e da Locomoção.

2 UFCG - Universidade Federal de Campina Grande - Centro de Formação de Professores de Cajazeiras.

Laboratório de Estudos da Postura e da Locomoção, Departamento de Educação Física, UNESP - Universidade Estadual Paulista -Campus Rio Claro - Avenida 24-A, 1515 - Bela Vista - 13.506-900 Rio Claro - São Paulo

Fone/Fax: (19) 3534-6436 - Email: 1ilian.gobbi@pq.cnpq.br 


\begin{abstract}
:
The locomotor behavior of 25 children (from two to six years old) was observed during walking on an obstacle circuit under normal and reduced illumination. Participants were instructed to walk as fast as possible avoiding the contact with the obstacles. Two dependent variables were collected: success rate and time spent to complete the task. The two-way ANOVAs ( 5 age groups) x (2 illumination), with repeated measures on the last factor revealed only group main effect for success rate $\left(\mathrm{F}_{4,20}=9.975 ; \mathrm{p}=0.001\right)$, lower for 2 years old children than for the other age groups; and for time spent to complete the task $\left(\mathrm{F}_{4,20}=3.136 ; \mathrm{p}=0.037\right)$, higher for 2 years old children than for 4 and 6 years old. Multiple regression analysis (stepwise) revealed that: chronological age, age group, walking experience, and time spent to complete the task predicted $75.3 \%$ of the success rate; and success rate was the only variable that predicted the spent time $\left(\mathrm{R}^{2}=0.372 ; \mathrm{p}=0.001\right)$. These results allowed us to conclude that developmental variables interfere on the locomotor behavior in an obstacle circuit and that illumination levels were not sufficient to disturb locomotion.
\end{abstract}

Key words: growth and development; perception; locomotion.

\section{INTRODUÇÃO}

Durante o período de desenvolvimento, muitos são os fatores que contribuem para a aquisição de habilidades motoras. Sendo o organismo um sistema complexo, em constante interação com o meio, toda ação motora será regida pelas restrições do organismo (peso, altura, comprimento dos segmentos corporais, entre outras) e do ambiente (clima, espaço físico, ação da gravidade e luminosidade, por exemplo), o que leva a criança à necessidade de adaptações constantes durante a realização de tarefas motoras. Estas adaptações passam a ser prospectivamente controladas à medida que a criança se desenvolve. ${ }^{1-4} \mathrm{O}$ resultado funcional das ações motoras está intrinsecamente ligado à realidade biomecânica do corpo da criança e às propriedades físicas do espaço onde se encontra. Portanto, o mesmo resultado funcional pode requerer uma ação motora diferente dependendo da condição do corpo. ${ }^{3,5,6}$

A análise da marcha tem sido largamente usada para o diagnóstico clínico de determinados distúrbios do movimento, bem como meio para se compreender o desenvolvimento motor durante o ciclo vital. ${ }^{7}$ A manutenção do equilíbrio durante a marcha envolve um relacionamento entre as forças que desestabilizam a propulsão do corpo para a frente e a necessidade de manter a estabilidade lateral do corpo, especialmente na fase de suporte simples. ${ }^{8}$

$\mathrm{O}$ andar é uma habilidade complexa que precisa ser adaptada ao ambiente, requerendo um elaborado controle sensoriomotor. Para manter o equilíbrio durante o andar, as informações visual, vestibular e somatosensorial detectam e modulam a ação motora no espaço pela posição relativa dos segmentos corporais. ${ }^{9}$ Além das restrições orgânicas decorrentes do rápido desenvolvimento nos primeiros anos de vida, a criança vive em um ambiente complexo, com obstáculos como mobiliários, degraus e outros objetos que são proporcionais à escala corporal dos adultos. Além disso, os sistemas perceptivo e motor da criança ainda não estão completamente maduros e integrados, o que dificulta a locomoção frente às irregularidades do terreno. ${ }^{10,11}$ A criança, então, necessita interagir com este ambiente para executar sua locomoção de forma segura e, para que isso ocorra, é necessário que ela consiga colher e integrar informações referentes ao organismo, ao ambiente e à tarefa..$^{5,9,10}$ 
Informações visuais sobre o ambiente e sobre o próprio indivíduo em relação ao ambiente são usadas para planejar o movimento humano. Para a transposição de um obstáculo no caminho durante a marcha, estas informações podem ser utilizadas tanto para o planejamento antecipatório da ação quanto durante a ação propriamente dita. ${ }^{10,12-14}$

Em estudos sobre o controle motor, os termos informação exteroceptiva (identificação visual da localização, das formas e superfícies existentes em determinado ambiente) e informação exproprioceptiva (identificação da relação entre os segmentos corporais e destes com os objetos e eventos no ambiente) são amplamente utilizados no sentido de oferecer recursos para a modulação ou adaptação do movimento durante o planejamento e realização de tarefa motora. ${ }^{10,12,14,15}$

Além disso, os movimentos coordenados envolvem otimização da interação entre forças musculares e forças passivas. Para a realização e o controle de movimentos habilidosos, o indivíduo não apenas necessita controlar as forças passivas, mas também explorar as propriedades mecânicas passivas para dirigir e aumentar o movimento. ${ }^{16}$ Esse controle é organizado a partir das informações proprioceptivas, em especial da cinestésica. Os sinais proprioceptivos informam sobre a posição e o movimento dos próprios membros sem o uso da visão. Existem duas categorias de informação proprioceptiva - a de posição estacionária dos membros (sentido e posição) e a de movimentação dos membros (cinestesia). ${ }^{17}$

A informação cinestésica é fornecida pelos receptores musculares e articulares que durante a locomoção em percursos com obstáculos enviam sinais sobre posição e velocidade da perna de suporte e de abordagem e a orientação do corpo em relação ao solo. ${ }^{10}$

Muitos são os estudos realizados em torno da questão perceptiva, principalmente com relação à visão durante a locomoção. O sistema visual fornece informações sobre as carac- terísticas do ambiente favorecendo a modulação do mecanismo de controle de padrões básicos do andar frente aos desafios encontrados no percurso. ${ }^{10,18}$ A marcha tem sido investigada sob diversas condições de visão, iluminação e com variação da tarefa, para análise das modulações do indivíduo durante a locomoção e também nas diferentes fases do desenvolvimento. ${ }^{11,19-21}$ Porém, são ainda insuficientes os estudos referentes ao desenvolvimento do andar em terrenos irregulares, ou com obstáculos.

Considerando a complexidade do terreno e a variação da luminosidade dos ambientes freqüentados pelas crianças diariamente, o objetivo deste estudo foi verificar o comportamento locomotor de crianças na faixa etária de dois a seis anos durante o andar em trajeto complexo e sob diferentes condições de iluminação.

\section{MÉTODO}

Participantes: 25 crianças, de ambos os sexos, na faixa etária de 2 a 6 anos, distribuídas em 5 grupos por idade (tabela 1), participaram deste estudo. Os pais e/ou responsáveis pelas crianças receberam informações sobre $o$ objetivo da pesquisa e os procedimentos experimentais. As coletas foram iniciadas após obtenção dos termos de consentimento fornecidos pelos pais.

Os dados apresentados na tabela 1 indicam que existe um aumento dos valores de G2 até G6 para todas as medidas antropométricas. Estes aumentos refletem o processo de crescimento rápido, mas pode-se perceber que os ganhos a cada ano são diferentes para cada medida antropométrica. Isto evidencia ritmos diferentes de crescimento segmentar.

Protocolo experimental: $O$ percurso era constituído de uma pista sinuosa, montada sobre um carpete medindo 7,10 m de comprimento por 1,00 m de largura, delimitada por balizas verticais medindo $62 \mathrm{~cm}$ de altura, interligadas por cordas de nylon. Dentro deste 
espaço delimitado foi montado um circuito onde foram posicionados 22 obstáculos de espuma (figura 1).

Os obstáculos variaram em comprimento, altura e largura de acordo com as características antropométricas dos participantes dos estudos de Soares ${ }^{22}$ e Lima. ${ }^{23}$ Para determinar os comprimentos, alturas e larguras dos obstáculos, foram usados os seguintes critérios: a altura do tornozelo contribuiu para a determi- nação das alturas dos obstáculos baixos; a altura do joelho possibilitou o estabelecimento das alturas dos obstáculos altos; e o comprimento do pé auxiliou nas dimensões dos obstáculos estreitos, correspondendo a $40 \%$ do comprimento do pé, e os largos, a $80 \%$.

$\mathrm{Na}$ parte externa do circuito foram colocados 2 holofotes de 500 watts cada e uma câmera filmadora. Os obstáculos foram posicionados no circuito de forma a garantir

Tabela 1: Caracterização da amostra. Valores de média e desvio-padrão de massa corporal (kg), estatura, comprimento da coxa, perna, altura do tornozelo e comprimento do pé (todos em $\mathrm{cm}$ ) e idade e experiência de andar (meses), quanto aos grupos (G2=2 anos; G3=3 anos; G4= 4 anos; $\mathrm{G} 5=5$ anos; G6 $=6$ anos).

\begin{tabular}{|c|c|c|c|c|c|}
\hline Características & $\begin{array}{c}\mathrm{G} 2(\mathrm{n}=5,19 \\
\text { a } 30 \text { meses })\end{array}$ & $\begin{array}{c}\mathrm{G} 3(\mathrm{n}=5,31 \\
\text { a } 42 \text { meses })\end{array}$ & $\begin{array}{c}\mathrm{G} 4(\mathrm{n}=5,43 \\
\text { a } 54 \text { meses })\end{array}$ & $\begin{array}{c}\mathrm{G5}(\mathrm{n}=5,55 \\
\text { a } 66 \text { meses })\end{array}$ & $\begin{array}{l}\mathrm{G6}(\mathrm{n}=5,67 \\
\text { a } 78 \text { meses })\end{array}$ \\
\hline Massa corporal & $12,04 \pm 1,06$ & $14 \pm 2,47$ & $16,9 \pm 1,75$ & $20,14 \pm 0,57$ & $21,64 \pm 4,58$ \\
\hline Estatura & $88,9 \pm 4,40$ & $95,1 \pm 4,96$ & $104,7 \pm 2,99$ & $111,7 \pm 2,56$ & $115 \pm 4,56$ \\
\hline Comprimento da coxa & $20 \pm 2,52$ & $21,2 \pm 0,4$ & $23 \pm 1,41$ & $24,8 \pm 1,93$ & $25 \pm 1,67$ \\
\hline Comprimento da perna & $19,5 \pm 1,84$ & $19,6 \pm 0,96$ & $23 \pm 1,78$ & $25,2 \pm 1,32$ & $27,2 \pm 0,74$ \\
\hline Altura do tornozelo & $3,6 \pm 0,48$ & $3,9 \pm 0,2$ & $4,4 \pm 0,48$ & $4,9 \pm 0,66$ & $5,4 \pm 0,48$ \\
\hline Comprimento do pé & $14 \pm 0,90$ & $14,26 \pm 0,75$ & $16,7 \pm 0,47$ & $17,9 \pm 1,00$ & $18,58 \pm 1,32$ \\
\hline Idade & $24,6 \pm 3,44$ & $38,2 \pm 1,93$ & $50,8 \pm 3,70$ & $62,8 \pm 0,97$ & $75,2 \pm 1,72$ \\
\hline Experiência de andar & $13,2 \pm 4,35$ & $25,6 \pm 2,33$ & $38,6 \pm 3,97$ & $50,6 \pm 2,60$ & $62,8 \pm 4,57$ \\
\hline
\end{tabular}

que variados graus de dificuldades fossem oferecidos para cada faixa etária, em diferentes pontos do circuito. A tabela 2 apresenta as dimensões dos obstáculos de acordo com as características antropométricas por grupo etário, cuja numeração corresponde à apresentada na figura 1.

Os participantes foram posicionados no início do circuito e instruídos a percorrê-lo andando o mais rápido possível evitando o contato com os obstáculos. Duas variáveis dependentes foram coletadas: tempo total para percorrer o circuito e taxa de sucesso. Para a coleta do tempo, ao sinal sonoro "já", emitido pelo experimentador, o cronômetro era disparado e só parado quando o participante cruzava as balizas verticais, posicionadas no final do circuito. A taxa de sucesso foi calculada através de porcentagem de acertos ao percorrer o circuito, sendo considerado como erro qualquer tipo de contato com os obstáculos e a não observância dos limites do circuito.

As crianças percorreram o circuito sob duas condições de iluminação, medidas através de luxímetro digital (Minipa MLM-1332). Condição 1: circuito iluminado, ou seja, os dois holofotes estavam com as lâmpadas ligadas (média de 114,21 \pm 15,08 LUX). Condição 2: circuito com iluminação reduzida, onde os dois holofotes estavam com as lâmpadas desliga- 
Figura 1: Esquema representativo da situação experimental.

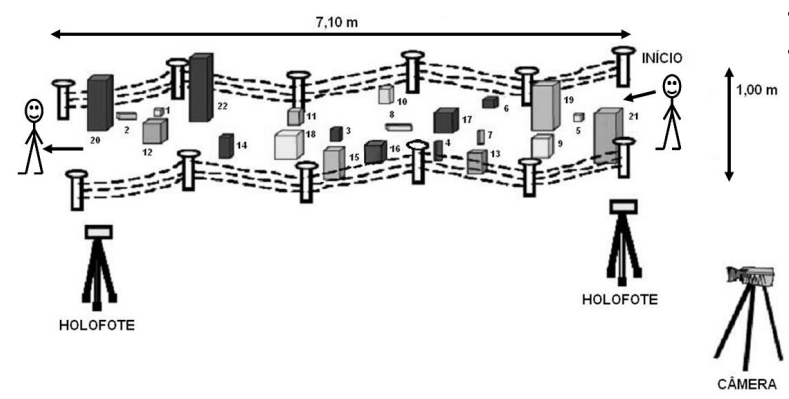

das (média de 70,79 \pm 14,67 LUX). Para cada condição de iluminação, três tentativas foram realizadas e a ordem de apresentação das condições de iluminação foi randomizada entre os participantes.

Análise dos dados: Os erros observados durante o trajeto e anotados na ficha de coleta foram confirmados pela revisão das imagens de cada participante. Para o cálculo da taxa de sucesso, considerou-se $100 \%$ de acerto os 22 obstáculos mais um acerto que era referente à finalização do trajeto respeitando a linha limite do circuito.

As médias e desvios-padrão da taxa de sucesso e do tempo gasto para percorrer o circuito foram calculados a partir dos dados de todas as tentativas válidas por grupo etário. Os dados de cada variável dependente foram tratados estatisticamente através da análise de variância, tendo grupo etário e iluminação como fatores, com medidas repetidas no último fator e nível de significância de $\mathrm{p} \leq 0,05$. As relações entre as variáveis antropométricas, desenvolvimentais e as varáveis dependentes foram tratadas estatisticamente através da análise de regressão múltipla (stepwise), para determinação das variáveis independentes que explicam a variabilidade da variável dependente, com o mesmo nível de significância. Em fuão do tamanho da amostra, optou-se por considerar a marginalidade da significância $\mathrm{p} \leq 0,10$.
Tabela 2: Dimensões dos obstáculos (altura e largura expressos em $\mathrm{cm}$ ).

\begin{tabular}{cccc}
\hline Obstáculos & Altura & Largura & Grupo etário \\
\hline 1 & 04 & 05 & $\mathrm{G} 2$ \\
2 & 04 & 08 & $\mathrm{G} 2$ \\
3 & 04 & 10 & $\mathrm{G} 2$ \\
4 & 04 & 15 & $\mathrm{G} 2$ \\
5 & 05 & 05 & $\mathrm{G} 3 / \mathrm{G} 4$ \\
6 & 05 & 08 & $\mathrm{G} 5 / \mathrm{G} 6$ \\
7 & 05 & 10 & $\mathrm{G} 3$ \\
8 & 05 & 15 & $\mathrm{G} 4 / \mathrm{G} 5 / \mathrm{G} 6$ \\
9 & 20 & 05 & $\mathrm{G} 2$ \\
10 & 20 & 08 & $\mathrm{G} 3$ \\
11 & 20 & 10 & $\mathrm{G} 2$ \\
12 & 20 & 15 & $\mathrm{G} 3$ \\
13 & 28 & 05 & $\mathrm{G} 4$ \\
14 & 28 & 08 & $\mathrm{G} 5 / \mathrm{G} 6$ \\
15 & 28 & 10 & $\mathrm{G} 4$ \\
16 & 28 & 15 & $\mathrm{G} 5 / \mathrm{G} 6$ \\
17 & 36 & 10 & $\mathrm{G} 2$ \\
18 & 36 & 15 & $\mathrm{G} 2$ \\
19 & 48 & 10 & $\mathrm{G} 3$ \\
20 & 48 & 15 & $\mathrm{G} 4$ \\
21 & 60 & 10 & $\mathrm{G} 5$ \\
22 & 60 & 15 & $\mathrm{G} 6$ \\
\hline
\end{tabular}

\section{RESULTADOS}

A ANOVA two-way (5 grupo-etário) x (2 iluminação), em relação à taxa de sucesso, com medidas repetidas no último fator, evidenciou efeito principal nos grupos etários $\left(\mathrm{F}_{4,20}=9,975 ; \mathrm{p}=0,001\right)$, marginalmente significante para iluminação $\left(\mathrm{F}_{1,20}=3,135\right.$; $\mathrm{p}=0,092)$ e interação não significativa entre grupo etário e iluminação. Na Figura $2 \mathrm{~A}$ se observa que G2 obteve taxa de sucesso inferior em relação aos demais grupos etários em ambas as condições de iluminação. Ainda, os valores percentuais da taxa de sucesso são superiores na condição de iluminação reduzida para G2, G3 e G6. Mesmo não havendo interação significativa, o teste "post-hoc" de 
Tukey, aplicado na taxa de sucesso, na condição com iluminação normal, evidenciou diferença do $\mathrm{G} 2$ em relação aos demais grupos, sendo G2 x G3 ( $\mathrm{p}=0,020), \mathrm{G} 2$ x G4 ( $\mathrm{p}=0,002)$ e $\mathrm{G} 2$ x G5 e G6 $(\mathrm{p}=0,001)$. Os valores percentuais médios obtidos pelo G2 na condição de iluminação normal foram inferiores em relação a todos os demais grupos etários. Na condição de iluminação reduzida, o teste "post-hoc" de Tukey evidenciou diferença significativa entre os grupos G2 x G5 ( $p=0,031)$, G2 x G6 (p=0,002). Na condição de iluminação reduzida, os valores percentuais da taxa de sucesso obtidos por G2 foram menores daqueles observados para os grupos mais velhos (G5 e G6).

A ANOVA two-way 5 (grupos) x 2 (iluminação), com medidas repetidas no último fator, revelou apenas efeito principal de grupo $\left(\mathrm{F}_{4,20}=3,136 ; \mathrm{p}=0,037\right)$ no tempo despendido para realizar a tarefa. Pode-se observar, na figura 2B, que os participantes de 4 e 6 anos apresentaram valores médios inferiores aos demais grupos, ou seja, percorreram o circuito em menor tempo. O G5 apresentou o maior desvio-padrão no tempo gasto para percorrer o

Figura 2: Representação gráfica dos valores percentuais médios e desvios-padrão da taxa de sucesso (A) e do tempo gasto (B) na realização da tarefa, por grupo etário e por condição de iluminação (CILU = com iluminação; ILUR = iluminação reduzida).

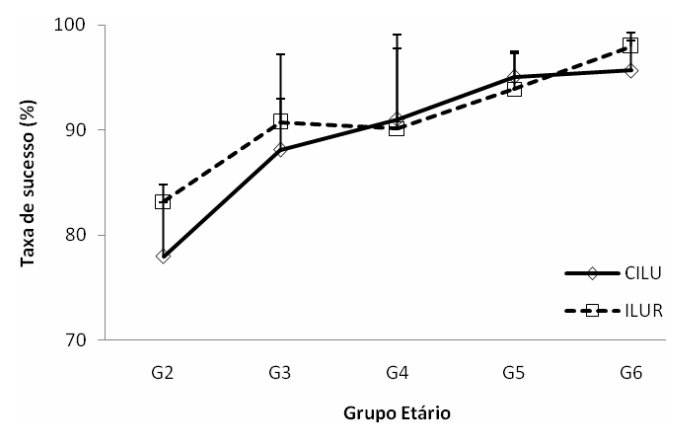

circuito. O teste "post-hoc" de Tukey, conduzido separadamente por condição de iluminação revelou que, apenas em condições de iluminação normal, as médias de G2 são estatisticamente diferentes de G4 $(p=0,021)$ e $\mathrm{G} 6(\mathrm{p}=0,007)$.

Uma Análise de regressão múltipla (stepwise) foi realizada para verificar as relações entre a taxa de sucesso e as variáveis antropométricas, desenvolvimentais e o tempo gasto para percorrer o circuito (tabela 3 ). Os resultados indicaram que idade cronológica, grupo etário, tempo de experiência de

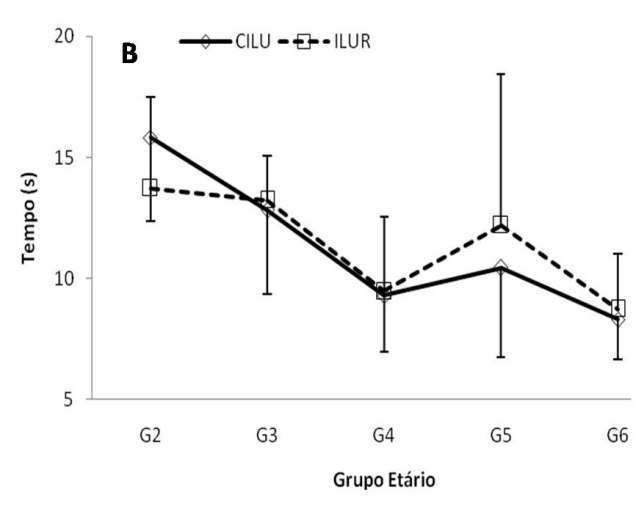

andar e tempo gasto para percorrer o circuito predizem $75,3 \%$ da taxa de sucesso. Contudo, as influências de cada variável dependente foram diferentes. A idade cronológica influenciou positivamente a taxa de sucesso, ou seja, à medida que a idade cronológica aumentou a taxa de sucesso também aumentou. Importante é considerar as contribuições das variáveis dependentes na variável independente como um todo. Idade cronológica, grupo etário, experiência de andar e taxa de sucesso se correlacionam positivamente. Desta forma, os valores negati- 
vos de Beta (tabela 3) indicam que a inclinação da curva altera-se, sem alterar o sentido positivo da reta ${ }^{24}$; visto que a idade cronológica, se considerada isoladamente, consegue predizer $60,5 \%$ da taxa de sucesso. Por outro lado, as crianças mais velhas executaram a tarefa em menor tempo.

A análise de regressão múltipla (stepwise) revelou que a taxa de sucesso foi a única variável que significativamente predisse o tempo despendido no circuito $\left(\mathrm{R}^{2}=0,372\right.$;
Beta $=-0,61 ; p=0,001 ;$ Figura 3). A taxa de sucesso interferiu negativamente no tempo para percorrer o circuito, ou seja, à medida que a taxa de sucesso aumentou, o tempo despendido diminuiu.

\section{DISCUSSÃO}

Em relação à iluminação, as diferenças da taxa de sucesso entre as condições de ilu-

Tabela 3: Variáveis preditoras da taxa de sucesso das crianças.

\begin{tabular}{cccc}
\hline VARIÁVEIS & $\mathbf{R}^{2}$ & BETA & SIGNIFICÂNCIA \\
\hline Idade & 0,605 & 4,734 & 0,000 \\
Grupo & 0,681 & $-2,088$ & 0,000 \\
Experiência de Andar & 0,722 & $-2,019$ & 0,008 \\
Tempo & 0,753 & $-0,215$ & 0,022 \\
\hline
\end{tabular}

Figura 3: Representação gráfica da taxa de sucesso como preditora do tempo gasto para percorrer o circuito.

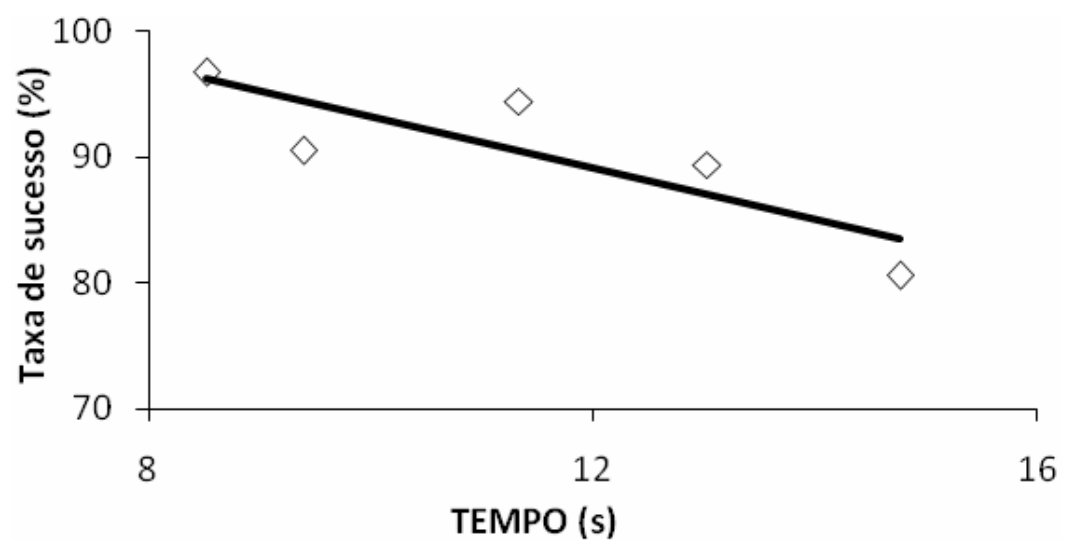

minação não são marcantes, porém, chegaram próximas aos níveis de significância para que esse fator fosse considerado perturbador para a realização da tarefa. Outros estudos, que usaram em seu protocolo diferentes taxas de iluminação, também não apresentaram resultados significativos em relação ao efeito da iluminação na realização de tarefa motora. ${ }^{11,25,26}$

Estudo sobre o papel da visão para transpor obstáculos, em adultos e crianças com média de 7 anos, sob alteração da luminosidade ambiente, evidenciou decréscimo da velocida- 
de e do comprimento do passo, e aumento na largura do passo. As crianças nesta idade apresentam forte influência e confiam na informação visual para guiar a colocação do pé em um ambiente complexo. ${ }^{27}$

A iluminação reduzida também evidenciou perturbação em indivíduos idosos. ${ }^{28} \mathrm{Nes}-$ te sentido, pode-se dizer que a taxa de $70 \mathrm{Lux}$ para a condição de iluminação reduzida utilizada no presente estudo não foi suficiente para perturbar a informação exproprioceptiva, importante para o sucesso e segurança da locomoção em terrenos complexos. ${ }^{29}$ Porém, devido à variedade de faixas etárias e aos níveis de iluminação nos protocolos utilizados nos estudos, novos estudos devem ser realizados.

Foram observados efeitos principais de grupo etário para a taxa de sucesso e para o tempo gasto para percorrer o circuito. As crianças mais velhas executaram a tarefa com menor quantidade de contatos e mais rapidamente que as crianças mais jovens. Estes resultados corroboram com os achados de Gobbi et al. ${ }^{11}$ que observaram crianças de 5 a 8 anos durante a locomoção em circuito com obstáculos. Neste estudo, os resultados demonstraram que as crianças de 5 e 6 anos foram semelhantes entre si, mas diferentes dos participantes das demais faixas etárias. A idade cronológica, portanto, evidenciou, no presente estudo, que quando variáveis de desempenho como tempo e erro são consideradas, as mudanças observadas indicam melhora no desempenho. ${ }^{30}$

Existe evidência sugerindo fortemente que o desempenho em equilíbrio de crianças pequenas está mais relacionado ao nível maturacional do que à idade cronológica. ${ }^{31}$ Crianças jovens não revelam o mesmo comportamento de crianças mais velhas, pois o desenvolvimento cognitivo, perceptivo-motor e da integração desses sistemas estão imaturos. ${ }^{11}$ Neste sentido, o presente estudo evidenciou que tanto a idade cronológica quanto a experiência de andar foram preditoras da taxa de sucesso.

Crianças com aproximadamente 12 meses de experiência do andar estão reduzindo a largura da passada e, conseqüentemente, reduzindo o movimento lateral do centro de massa indicando melhor controle do equilíbrio. ${ }^{9}$ Entretanto, a média de 13,2 meses de experiência do andar das crianças do G2 não mostrou ser suficiente para o sucesso na realização da tarefa. As crianças deste grupo apresentaram dificuldades para a locomoção no circuito, indicadas pela menor taxa de sucesso e pelo maior tempo gasto no percurso.

Neste sentido, estudos específicos sobre o tempo de experiência de andar e a eficiência da marcha indicam que crianças de até 3 anos de idade apresentam alta variabilidade de ativação muscular durante a marcha, com pouco ou nenhum padrão de comportamento. A cocontração muscular diminui com a experiência de andar, porém permanece inconsistente enquanto a estabilidade da marcha aumenta significativamente, o que pode indicar que crianças nesta faixa etária têm força suficiente para estabilizar e deslocar, no entanto, a descoberta de como otimizar o movimento acontece lentamente. ${ }^{1,4}$

Sutherland ${ }^{32}$, em estudo sobre os aspectos desenvolvimentais da marcha em diferentes idades, encontrou que crianças de até 3 anos de idade, observadas no plano coronal, apresentam pequena elevação do quadril do membro ipsilateral, acompanhada de menor adução do quadril na fase de apoio durante a locomoção. Embora a avaliação do presente estudo não tenha sido realizada a partir do plano coronal, as evidências de imaturidade do controle da cintura pélvica, descritas por Sutherland ${ }^{32}$, podem ter contribuído para os valores da taxa de sucesso nas crianças do G2.

Quanto ao tempo gasto, também na condição de iluminação normal, G2 realizou a tarefa mais lentamente que G4 e G6. Estes re- 
sultados revelam que as crianças do G2 são imaturas na experiência do andar, pois a musculatura flexora do quadril ainda é frágil e a base de suporte ainda continua aberta ${ }^{6}$, dificultando a ultrapassagem sobre obstáculos. Além disso, a amplitude dinâmica na articulação do quadril é 9 graus menor em crianças de 1 e 2 anos quando comparadas com crianças de 3 a 7 anos. ${ }^{31}$ A estabilização da pélvis auxilia o controle médio-lateral do centro de gravidade do corpo. Esta estabilização tem sido mostrada como uma ativação antecipatória do abdutor do quadril da perna de suporte antes da retirada do calcanhar do solo. ${ }^{33}$ Neste experimento, o deslocamento médio-lateral foi requerido para a realização da tarefa.

Ao observar as filmagens, constatou-se que as crianças do G5 e G6 criaram estratégias inteligentes para não tocarem os obstáculos durante o trajeto como, por exemplo, levantar os braços ou segurar na roupa. Estas posturas dos braços, congelando graus de liberdade, aumentam as forças oriundas da inclinação pélvica tornando a postura menos estável durante o andar. ${ }^{34}$ Por outro lado, crianças por volta de 5 e 6 anos exploram novas estratégias locomotoras, as quais podem ser sensíveis a qualquer tipo de perturbação do ambiente. ${ }^{9}$ Os resultados de G5 confirmam essas afirmações, especialmente quanto ao tempo gasto para percorrer o circuito, pois seus valores médios foram superiores e mais variáveis do que G4 e G6.

A lei de Fitts ${ }^{35}$ aparentemente não explica as relações entre taxa de sucesso e tem-

\section{REFERÊNCIAS}

1. Chang C-L, Kubo M, Buzzi U, Ulrich B. Early changes in muscle activation patterns of toddlers during walking. Infant Behavior and Development. 2006; 29(2): 175-88.

2. Joh AS, Adolph KE. Learning from falling. Child Dev. 2006; 77(1): 89-102. po gasto para percorrer o circuito. De acordo com esta lei, existe uma relação inversamente proporcional entre precisão e velocidade durante a execução da ação motora. ${ }^{35,36}$ No presente estudo, quanto mais rápido as crianças executaram a tarefa, maior foi a taxa de sucesso observada.

A locomoção em circuito com obstáculos requereu dos participantes a percepção dos objetos e a modulação do sistema efetor através da elaboração de estratégias motoras que apresentaram variações de uma idade à outra sendo que, quanto maior a idade, mais elaboradas se mostravam essas estratégias. Entretanto, novos estudos devem ser delineados aplicando-se maior restrição de iluminação no circuito de obstáculos; incluindo obstáculos suspensos; utilizando outras tarefas que requeiram reversão ou finalização no sentido do movimento e observando longitudinalmente o desempenho das crianças.

Assim, observa-se que a idade cronológica influencia no desempenho locomotor de crianças entre dois e seis anos de idade, mas que variáveis desenvolvimentais interferem diferentemente no desempenho locomotor em ambiente complexo. Ainda, a luminosidade do ambiente, nos limites empregados neste estudo, não interfere de forma efetiva na realização de tarefa locomotora. As variáveis antropométricas não interferem na locomoção sobre obstáculos quando estes são personalizados.

3. Holt KG, Saltzman E, Ho C-L, Kubo M, Ulrich BD. Discovery of the pendulum and spring dynamics in the early stages of walking. J Motor Behav. 2006; 38(3): 206-18.

4. Holt KG, Saltzman E, Ho C-L, Ulrich BD. Scaling of dynamics in the earliest stages of walking. Phys Ther. 2007; 87(11): 1458-67. 
5. Haywood KM, Getchell N. Desenvolvimento motor ao longo da vida. 3 ed. Porto Alegre: Artmed; 2004.

6. Adolph KE, Berger SA. Motor development. In: Damon W, Lerner R, series editors; Kuhn D, Siegler RS, volume editors. Handbook of child psychology: cognition, perception, and language. 6th ed. New York: Wiley; 2006; v. 2. p. 161-213.

7. Leardini A, Sawacha Z, Paolini G, Ingrosso S, Nativo R, Benedetti MG. A new anatomically based protocol for gait analysis in children. Gait Posture. 2007; 26(4): 560-71.

8. Winter DA. Biomechanics and Motor Control of Human Movement. 2nd ed. New York: Wiley-Interscience; 1990.

9. Bril B, Ledebt A. Head coordination as a mean to assist sensory integration in learning to walk. Neurosci Biobehav Rev. 1998; 22(4): 555-63.

10. Gobbi LTB, Patla AE. Desenvolvimento da locomoção em terrenos irregulares: proposta de um modelo teórico. In: Pellegrini AM, organização. Coletânea de Estudos: comportamento motor I. São Paulo: Movimento; 1997. p. 29-44.

11. Gobbi LTB, Silva JJ, Paiva ACS, Scabello PL. Comportamento locomotor de crianças e adultos jovens em ambiente doméstico simulado. Psic: Teor e Pesq. 2007; 23(3): 273-8.

12. Patla AE. Understanding the roles of vision in the control of human locomotion. Gait Posture. 1997; 5(1): 54-69.

13. Heath M, Rival C, Neely K, Krigolson O. Müller-Lyer figures influence the online reorganization of visually guided grasping movements. Exp Brain Res. 2006; 169(4): 473-81.
14. Rhea CK, Rietdyk S. Visual exteroceptive information provided during obstacle crossing did not modify the lower limb trajectory. Neurosci Lett. 2007; 418(1): 60-5.

15. Mohagheghi AA, Moraes R, Patla AE. The effects of distant and on-line visual information on the control of approach phase and step over an obstacle during locomotion. Exp Brain Res. 2004; 155(4): 459-68.

16. Zernicke RF, Schneider K, Buford JA. Intersegmental Dynamics During Gait: Implications for Control. In: Patla AE. Adaptability of Human Gait. NorthHolland: Elsevier Science Publishers BV; 1991. p. 187-202.

17. Kandel ER, Schwartz JH, Jessell TM. As Sensações Corporais. In: Kandel ER, Schwartz JH, Jessell TM. Princípios da Neurociência. 4 ed. Baruerí, SP: Manole; 2003. p. 430-50.

18. Patla AE, Vickers JN. How far ahead do we look when required to step on specific locations in the travel path during locomotion? Exp Brain Res. 2003; 148(1): 133-8.

19. Lu TW, Chen HL, Chen SC. Comparisons of the lower limb kinematics between young and older adults when crossing obstacles of different heights. Gait Posture. 2006; 23(4): 471-9.

20. Jordan K, Challis JH, Newell KM. Walking speed influences on gait cycle variability. Gait Posture. 2007; 26: 128-34.

21. Fuller JR, Adkin AL, Vallis LA. Strategies used by older adults to change travel direction. Gait Posture. 2007; 25: 393-400. 
22. Soares IC. Efeitos da natação de bebês na locomoção em ambiente complexo [trabalho de conclusão de curso]. Rio Claro, SP: Instituto de Biociências Universidade Estadual Paulista; 1997.

23. Lima CB. Equilíbrio dinâmico: influência das restrições ambientais [trabalho de conclusão de curso]. Rio Claro, SP: Instituto de Biociências Universidade Estadual Paulista; 1999.

24. Cohen J, Cohen P. Applied Multiple Regression/Correlation Analysis for the Behavioral Sciences. 2nd ed. Hillsdale: Lawrence; 1983.

25. Paiva ACS, Gobbi LTB. Efeitos da experiência motora no desempenho locomotor em tarefa da vida diária [resumo expandido]. In: Anais do II Seminário De Comportamento Motor. São Paulo: Laboratório de Comportamento Motor - Faculdade de Educação Física e Esportes da USP; 2000. p. 126.

26. Blanchard $Y$, Mcveigh R, Graham M, Cadet M, Mwilambwe K, Scott C. The influence of ambient lighting levels on postural sway in healthy children. Gait Posture. 2007; 26(3): 442-5.

27. Berard JR, Vallis LA. Characteristics of single and double obstacle avoidance. Exp Brain Res. 2006; 175(1): 21-31.

28. Elliott DB, Patla AE, Furniss M, Adkin A. Improvements in clinical and functional vision and quality of life after second eye cataract surgery. Optom Vis Sci. 2000; 77(1): 13-24.
29. Gobbi LTB, Menuchi MRTP, Uehara ET, Silva JJ. Influência da informação exproprioceptiva em tarefa locomotora com alta demanda de equilíbrio em crianças. Revista Brasileira de Ciência e Movimento. 2003; 11(4): 79-86.

30. Clark JE, Whitall J. What is motor development? The lessons of history. Quest. 1989; 41(3): 183-202.

31. Gabbard C. Lifelong Motor Development. 3rd ed. San Francisco: Benjamin Cummings, 2000.

32. Sutherland D. The development of mature gait. Gait Posture. 1997; 6(2): 163-70.

33. Assaiante C. Development of locomotor balance control in healthy children. Neurosci Biobehav Rev. 1998; 22(4): 527-32.

34. Ledebt A. Changes in arm posture during the early acquisition of walking. Infant Behavior and Development. 2000; 23(1): 79-89.

35. Fitts PM. Cognitive Aspects of Information Processing: III. Set for speed versus accuracy. J Exp Psychol. 1966; 71(6): 849-57.

36. Schmidt RA, Wrisberg CA. Motor Learning and Performance: a problembased learning approach. Champaign: Human Kinetics; 2000. 338 p.

Recebido em: 06/10/2008 Modificado em: 10/11/2008 Aceito em: 22/11/2008 